\title{
Common Random Fixed Point and Random Best Approximation in Non-Starshaped Domain of $q$-Normed Spaces
}

\author{
Hemant Kumar Nashine
}

\begin{abstract}
The aim of this paper is to obtain some common random fixed point by extending the concept of uniformly $\mathcal{R}$-subweakly commuting mappings to the case of non-starshaped domain in $q$-normed space. Random best approximation results have also been obtained as its application. This work provides extension as well as substantial improvement of some results in the existing literature.
\end{abstract}

\section{Introduction AND PRELIMINARIES}

In this paper the following definitions have been used:

Definition 1.1. [8]. Let $(\Omega, \mathcal{A})$ be a measurable space and $\mathcal{X}$ be a metric space. Let $2^{\mathcal{X}}$ be the family of all nonempty subsets of $\mathcal{X}$ and $\mathcal{C}(\mathcal{X})$ denote the family of all nonempty compact subsets of $\mathcal{X}$. A mapping $\mathcal{T}: \Omega \rightarrow$ $2^{\mathcal{X}}$ is called measurable (respectively, weakly measurable) if, for any closed (respectively, open) subset $\mathcal{B}$ of $\mathcal{X}, \mathcal{T}^{-1}(\mathcal{B})=\{\omega \in \Omega: \mathcal{T}(\omega) \cap \mathcal{B} \neq \emptyset\} \in \mathcal{A}$. Note that, if $\mathcal{T}(\omega) \in \mathcal{C}(\mathcal{X})$ for every $\omega \in \Omega$, then $\mathcal{T}$ is weakly measurable if and only if measurable.

A mapping $\xi: \Omega \rightarrow \mathcal{X}$ is said to be measurable selector of a measurable mapping $\mathcal{T}: \Omega \rightarrow 2^{\mathcal{X}}$, if $\xi$ is measurable and, for any $\omega \in \Omega, \xi(\omega) \in \mathcal{T}(\omega)$. A mapping $\mathcal{T}: \Omega \times \mathcal{X} \rightarrow \mathcal{X}$ is called a random operator if, for any $x \in \mathcal{X}$, $\mathcal{T}(., x)$ is measurable. A measurable mapping $\xi: \Omega \rightarrow \mathcal{X}$ is called a random fixed point of a random operator $\mathcal{T}: \Omega \times \mathcal{X} \rightarrow \mathcal{X}$, if for every $\omega \in \Omega$, $\xi(\omega)=\mathcal{T}(\omega, \xi(\omega))$.

For the detail of $q$-normed space, we refer the reader to Köthe [13] and Rudin [21, page 36 and 37].

Let $\mathcal{X}$ be a $q$-normed space. A map $\mathcal{T}: \mathcal{X} \rightarrow \mathcal{X}$ is said to be

2000 Mathematics Subject Classification. Primary: 41A50; Secondary: 41A65, 47H10, $60 \mathrm{H} 25$.

Key words and phrases. Asymptotically nonexpansive maps, Uniformly asymptotically regular map, Random best approximation, Random fixed point, Random operator, Nonexpansive mapping, Uniformly $\mathcal{R}$-subweakly commuting maps, Property $(N)$, Property $(C), q$-normed space. 
(1) a uniformly asymptotically regular on $\mathcal{X}$ if, for each $\eta>0$, there exists $N(\eta)=N$ such that $\left\|\mathcal{T}_{n} x-\mathcal{T}_{n+1} x\right\|_{q}<\eta$ for all $\eta \geq 0$ and all $x \in \mathcal{X}$.

(2) $\mathcal{S}$-nonexpansive, if there exists a self-map $\mathcal{S}$ on $\mathcal{X}$ such that

$$
\|\mathcal{T} x-\mathcal{T} y\|_{q} \leq\|\mathcal{S} x-\mathcal{S} y\|_{q} \text { for all } x, y \in \mathcal{X}
$$

(3) asymptotically $\mathcal{S}$-nonexpansive, if there exists a sequence $\left\{k_{n}\right\}$ of real numbers with $k_{n} \geq 1$ and $\lim _{n \rightarrow \infty} k_{n}=1$ such that $\left\|\mathcal{T}^{n} x-\mathcal{T}^{n} y\right\|_{q} \leq$ $\left(k_{n}\right)^{q}\|\mathcal{S} x-\mathcal{S} y\|_{q}$ for all $x, y \in \mathcal{X}$ and $n=1,2,3, . . \infty$.

Two maps $\mathcal{T}, \mathcal{S}: \mathcal{X} \rightarrow \mathcal{X}$ are called

(4) $\mathcal{R}$-weakly commuting, if there exists some $\mathcal{R}>0$ such that

$$
\|\mathcal{T} \mathcal{S} x-\mathcal{S} \mathcal{T} x\|_{q} \leq \mathcal{R}\|\mathcal{T} x-\mathcal{S} x\|_{q} \quad \text { for all } \quad x \in \mathcal{X}
$$

Suppose $p \in \operatorname{Fix}(\mathcal{S}), \mathcal{M} \subset \mathcal{X}$ is $p$-starshaped and both $\mathcal{T}$ and $\mathcal{S}$ are invariant. Then $\mathcal{T}$ and $\mathcal{S}$ are said to be

(5) $\mathcal{R}$-subweakly commutating on $\mathcal{M}$, if there exists a real number $\mathcal{R}>0$ such that

$$
\|\mathcal{T} \mathcal{S} x-\mathcal{S} \mathcal{T} x\|_{q} \leq \mathcal{R} \operatorname{dist}(\mathcal{S} x,[\mathcal{T} x, p])
$$

for all $x \in \mathcal{M}$ where $\operatorname{dist}(\mathcal{S} x,[\mathcal{T} x, p])=\inf \{\|\mathcal{S} x-z\|: z \in[\mathcal{T} x, p]\}$ and $[\mathcal{T} x, p]=\{k \mathcal{T} x+(1-k) p: 0 \leq k \leq 1\}$. Obviously, commutativity implies $\mathcal{R}$-subweak commutativity but the converse is not true in general [23].

(6) uniformly $\mathcal{R}$-subweakly commuting on $\mathcal{M}-\{p\}$ if there exists a real number $\mathcal{R}>0$ such that

$$
\left\|\mathcal{T}^{n} \mathcal{S} x-\mathcal{S} \mathcal{T}^{n} x\right\|_{q} \leq \mathcal{R} \operatorname{dist}\left(\mathcal{S} x,\left[\mathcal{T}^{n} x, p\right]\right)
$$

for all $x \in \mathcal{M}-\{p\}$ and $n \in N[2]$.

It is clear from (6) that uniformly $\mathcal{R}$-subweakly commuting mappings on $\mathcal{M}-\{p\}$ are $\mathcal{R}$-subweakly commuting on $\mathcal{M}-\{p\}$, but $\mathcal{R}$-subweakly commuting mappings on $\mathcal{M}-\{p\}$ need not be uniformly $\mathcal{R}$-subweakly commuting on $\mathcal{M}-\{p\}$.

A random operator $\mathcal{T}: \Omega \times \mathcal{X} \rightarrow \mathcal{X}$ is continuous (respectively, nonexpansive, $\mathcal{S}$-nonexpansive) if, for each $\omega \in \Omega, \mathcal{T}(\omega,$.$) is continuous (respectively,$ nonexpansive, $\mathcal{S}$-nonexpansive). Random operators $\mathcal{T}, \mathcal{S}: \Omega \times \mathcal{X} \rightarrow \mathcal{X}$ are $\mathcal{R}$-weakly commuting (respectively $\mathcal{R}$-subweakly commuting, uniformly $\mathcal{R}$-subweakly commuting), if $\mathcal{T}(\omega,$.$) and \mathcal{S}(\omega,$.$) are \mathcal{R}$-weakly commuting (respectively $\mathcal{R}$-subweakly commuting, uniformly $\mathcal{R}$-subweakly commuting) for each $\omega \in \Omega$.

Definition 1.2. Let $\mathcal{X}$ be a complete $q$-normed space whose dual $\mathcal{X}^{\prime}$ separates the points of $\mathcal{X}$ and $\mathcal{M}$ be a subset of $\mathcal{X}$. The map $\mathcal{T}: \mathcal{M} \rightarrow \mathcal{X}$ is said to be demiclosed at 0 if for every sequence $\left\{x_{n}\right\}$ in $\mathcal{M}$ such that $\left\{x_{n}\right\}$ 
converges weakly to $x$ and $\left\{\mathcal{T} x_{n}\right\}$ converges strongly to $0 \in \mathcal{X}$, then $0=\mathcal{T} x$. The space $\mathcal{X}$ is said to satisfy Opial's condition if for every sequence $\left\{x_{n}\right\}$ in $\mathcal{X}$ weakly convergent to $x \in \mathcal{X}$, the inequality $\liminf _{n \rightarrow \infty}\left\|x_{n}-x\right\|_{q}<$ $\operatorname{limin} f_{n \rightarrow \infty}\left\|x_{n}-y\right\|_{q}$ holds for all $y \neq x$. Every Hilbert space and the space $l_{q}(1 \leq q<\infty)$ satisfy Opial's condition.

We now introduce the property $(N)$ and $(C)$ [12].

Definition 1.3. A set $\mathcal{M}$ is said to have property $(N)$ if

(i) $\mathcal{T}: \mathcal{M} \rightarrow \mathcal{M}$,

(ii) $\left(1-\mu_{n}\right) p+\mu_{n} \mathcal{T}^{n} x \in \mathcal{M}$, for some $p \in \mathcal{M}$ and a fixed sequence of real numbers $\mu_{n}\left(0<\mu_{n}<1\right)$ converging to 1 and for each $x \in \mathcal{M}$.

A mapping $\mathcal{S}$ is said to have property $(C)$ on a set $\mathcal{M}$ with the property $(N)$ if $\mathcal{S}\left(\left(1-\mu_{n}\right) p+\mu_{n} \mathcal{T}^{n} x\right)=\left(1-\mu_{n}\right) \mathcal{S} p+\mu_{n} \mathcal{S} \mathcal{T}^{n} x$ for each $x \in \mathcal{M}$ and $n \in N$.

We extend the concept of uniformly $\mathcal{R}$-subcommuting maps to non-starshaped domain in the following way:

Let $\mathcal{S}$ and $\mathcal{T}$ be self-maps on the set $\mathcal{M}$ having property $(N)$ with $p \in \operatorname{Fix}(\mathcal{S})$. Then $\mathcal{S}$ and $\mathcal{T}$ are called uniformly $\mathcal{R}$-subweakly commuting on $\mathcal{M}-\{p\}$, provided for all $x \in \mathcal{M}$, there exists a real number $\mathcal{R}>0$ such that

$$
\left\|\mathcal{T}^{n} \mathcal{S} x-\mathcal{S} \mathcal{T}^{n} x\right\|_{q} \leq \mathcal{R} \operatorname{dist}\left(\mathcal{S} x,\left[\mathcal{T}^{n} x, p\right]\right)
$$

for all $x \in \mathcal{M}-\{p\}$, where $\left[\mathcal{T}^{n} x, p\right]=\left(1-\mu_{n}\right) p+\mu_{n} \mathcal{T}^{n} x$, and $\left\{\mu_{n}\right\}$ is a sequence of real numbers with $\mu_{n} \in[0,1]$ and $\lim _{n \rightarrow \infty} \mu_{n}=1$. Each $\mathcal{T}$ invariant $p$-starshaped set has property $(N)$ but not conversely in general. Each affine map on a $p$-starshaped set $\mathcal{M}$ satisfies property $(C)$.

Definition 1.4. [8]. Let $\mathcal{M}$ be a nonempty subset of a $q$-normed space $\mathcal{X}$. For $x_{0} \in \mathcal{X}$, let us define

$$
\operatorname{dist}\left(x_{0}, \mathcal{M}\right)=\inf _{y \in \mathcal{M}}\left\|x_{0}-y\right\|_{q}
$$

and

$$
\mathcal{P}_{\mathcal{M}}\left(x_{0}\right)=\left\{y \in \mathcal{M}:\left\|x_{0}-y\right\|_{q}=\operatorname{dist}\left(x_{0}, \mathcal{M}\right)\right\} .
$$

An element $y \in \mathcal{P}_{\mathcal{M}}\left(x_{0}\right)$ is called a best approximant of $x_{0}$ out of $\mathcal{M}$. The set $\mathcal{P}_{\mathcal{M}}\left(x_{0}\right)$ is the set of all best approximation of $x_{0}$ out of $\mathcal{M}$.

Probabilistic functional analysis is an important mathematical discipline because of its applications to probabilistic models in applied problems. Random operator theory is needed for the study of various classes of random equations. The theory of random fixed point theorems was initiated by the Prague school of probabilistic in 1950s. The interest in this subject enhanced after publication of the survey paper by Bharucha Reid [9]. Random fixed point theory has received much attention in recent years(see [4, 19, 20, 25]).

Random fixed point theorems and random approximations are stochastic generalization of classical fixed point and approximation theorems, and have 
application in probability theory and nonlinear analysis. The random fixed point theory for self-maps and non-self maps has been developed during the last decade by various author, (see e.g. $[3,4,11]$ ). Recently, this theory has been further extended for 1-set contractive, nonexpansive, semi-contractive and completely continuous random maps, etc.

Random fixed point theorems have been applied in many instances in the field of random best approximation theory and several interesting and meaningful results have been studied. The theory of approximation has become so vast that it intersects with every other branch of analysis and plays an important role in the applied sciences and engineering. Approximation theory is concerned with the approximation of functions of a certain kind by other functions. In this perspective, in the year 1963, Meinardus [16] was first to observe the general principle and to use a Schauder fixed point theorem. Later on, number of results were developed in this direction under different conditions following the line made by Meinardus (see $[3,4,6,8]$ ).

In the year 1996, Beg and Shahzad [6] gave the random version of invariant approximation for the pair of commuting linear mapping for compact and starshaped subset of normed space. Subsequently, in the year 2000, Beg and Shahzad [8] gave the random version of invariant approximation for pair of noncommuting linear mapping for compact and starshaped subset of Banach space. It was further improved and extended by Nashine [18] by considering $\mathcal{R}$-subweakly commuting and uniformly $\mathcal{R}$-subweakly commuting maps on the starshaped domain and affinity of random operators $\mathcal{S}$.

Attempt has been made to extend the results of Nashine [18] by extending the concept of uniformly $\mathcal{R}$-subweakly commuting mappings to the case of non-starshaped domain and relaxing the affinity of random operators $\mathcal{S}$ in $q$-normed space. As application, some invariant approximation results have also been determined. Incidently, results of Beg and Shahzad [6, Theorem 2], Beg and Shahzad [8, Theorem B] and Nashine [17] are improve and generalized with the aid of more general class of noncommuting random operators and relaxing the condition of linearity of random operators $\mathcal{S}$. Our results also give stochastic version generalization of common fixed point theorem of Dotson [10] and invariant approximation theorem of Sahab et al. [22], Singh [24] and Shahzad [23].

The following result is needed in the sequel:

Theorem $1.1([18])$. Let $\mathcal{M}$ be a subset of a q-normed space $\mathcal{X}$ and $\mathcal{T}, \mathcal{S}$ : $\Omega \times \mathcal{M} \rightarrow \mathcal{M}$ be two random operators such that, for each $\omega \in \Omega, \mathcal{T}(\omega, \mathcal{M}-$ $\{p\}) \subseteq \mathcal{S}(\omega, \mathcal{M}-\{p\})$ where $p \in F i x(\mathcal{S})$. Suppose $\mathcal{T}$ is continuous and

$$
d(\mathcal{T}(\omega, x), \mathcal{T}(\omega, y)) \leq k(\omega) d(\mathcal{S}(\omega, x), \mathcal{S}(\omega, y))
$$

for all $x, y \in \mathcal{M}, \omega \in \Omega$ and $k(\omega) \in(0,1)$ such that $\mathcal{S}(\omega, x) \neq \mathcal{S}(\omega, y)$. If $\mathcal{T}$ and $\mathcal{S}$ are $\mathcal{R}$-weakly commutative on $\mathcal{M}-\{p\}$, then $\mathcal{T}$ and $\mathcal{S}$ have a unique common random fixed point. 


\section{MAIN RESULTS}

Following result is extension of Theorem 3.4 [18] in the case of nonstarshaped domain of $q$-normed space:

Theorem 2.1. Let $\mathcal{M}$ be a nonempty compact subset of a q-normed space $\mathcal{X}$ and let $\mathcal{T}, \mathcal{S}: \Omega \times \mathcal{X} \rightarrow \mathcal{X}$ be uniformly $\mathcal{R}$-subweakly commutative random operators on $\mathcal{M}-\{p\}$ such that for each $\omega \in \Omega$. Assume that $\mathcal{M}$ has the property $(N), \mathcal{S}(\omega, \mathcal{M})=\mathcal{M}$ and $\mathcal{T}(\omega, \mathcal{M}-\{p\}) \subseteq \mathcal{S}(\omega, \mathcal{M}-\{p\})$ where $p \in F i x(\mathcal{S})$. Suppose $\mathcal{T}$ is continuous, asymptotically $\mathcal{S}$-nonexpansive with sequence $\left\{k_{n}\right\}$ and $\mathcal{S}(\omega,$.$) satisfies property (C)$. For each $n \geq 1$, define a random operator $\mathcal{T}_{n}(\omega,$.$) by \mathcal{T}_{n}(\omega, x)=\mu_{n}(\omega) \mathcal{T}^{n}(\omega, x)+\left(1-\mu_{n}(\omega)\right) p, x \in$ $\mathcal{M}$, where $\mu_{n}(\omega)=\frac{\lambda_{n}(\omega)}{k_{n}(\omega)}$ and $\lambda_{n}(\omega) \in(0,1)$ such that $\lim _{n \rightarrow \infty} \lambda_{n}(\omega)=1$. Then for each $n \geq 1, \mathcal{T}_{n}$ and $\mathcal{S}$ have exactly one common random fixed point.

Proof. For all $x, y \in \mathcal{M}$, we have

$$
\begin{aligned}
\left\|\mathcal{T}_{n}(\omega, x)-\mathcal{T}_{n}(\omega, y)\right\|_{q} & =\left[\mu_{n}(\omega)\right]^{q}\left\|\mathcal{T}^{n}(\omega, x)-\mathcal{T}^{n}(\omega, y)\right\|_{q} \\
& \leq\left[\lambda_{n}(\omega)\right]^{q}\|\mathcal{S}(\omega, x)-\mathcal{S}(\omega, y)\|_{q} .
\end{aligned}
$$

Also, $\mathcal{T}_{n}$ is a self-mapping of $\mathcal{M}$ such that $\mathcal{T}_{n}(\mathcal{M}-\{p\}) \subseteq \mathcal{S}(\mathcal{M}-\{p\})$ for each $n$. From the uniformly $\mathcal{R}$-subweakly commutativity of $\mathcal{S}$ and $\mathcal{T}$ on $\mathcal{M}-\{p\}$ and property $(C)$ of $\mathcal{S}$, it follows that

$$
\begin{aligned}
& \left\|\mathcal{T}_{n}(\omega, \mathcal{S}(\omega, x))-\mathcal{S}\left(\omega, \mathcal{T}_{n}(\omega, x)\right)\right\|_{q} \\
= & \| \mu_{n}(\omega) \mathcal{T}^{n}(\omega, \mathcal{S}(\omega, x))+\left(1-\mu_{n}(\omega)\right) p- \\
& \quad-\mathcal{S}\left(\omega, \mu_{n}(\omega) \mathcal{T}^{n} x+\left(1-\mu_{n}(\omega) p\right)\right) \|_{q} \\
= & {\left[\mu_{n}(\omega)\right]^{q}\left\|\mathcal{T}^{n}(\omega, \mathcal{S}(\omega, x))-\mathcal{S}\left(\omega, \mathcal{T}^{n}(\omega, x)\right)\right\|_{q} } \\
\leq & {\left[\mu_{n}(\omega)\right]^{q} \mathcal{R} \operatorname{dist}\left(\mathcal{S}(\omega, x),\left[\mathcal{T}^{n}(\omega, x), p\right]\right) } \\
\leq & {\left[\mu_{n}(\omega)\right]^{q} \mathcal{R}\left\|\mu_{n}(\omega) \mathcal{T}^{n}(\omega, x)+\left(1-\mu_{n}(\omega)\right) p-\mathcal{S}(\omega, x)\right\|_{q} } \\
\leq & {\left[\mu_{n}(\omega)\right]^{q} \mathcal{R}\left\|\mathcal{T}_{n}(\omega, x)-\mathcal{S}(\omega, x)\right\|_{q} }
\end{aligned}
$$

for all $x \in \mathcal{M}-\{p\}$. Thus $\mathcal{T}_{n}$ and $\mathcal{S}$ are $\left[\mu_{n}(\omega)\right]^{q} \mathcal{R}$-weakly commuting. Therefore, Theorem 1.1 implies that there exists a random fixed point $\xi_{n}(\omega)$ of $\mathcal{T}_{n}$ and $\mathcal{S}$ such that $\xi_{n}(\omega)=\mathcal{S}\left(\omega, \xi_{n}(\omega)\right)=\mathcal{T}_{n}\left(\omega, \xi_{n}(\omega)\right)$ for each $\omega \in \Omega$.

The following theorem is the common random fixed point results for uniformly $\mathcal{R}$-subweakly commuting mappings in the case of non-starshaped domain of $q$-normed space:

Theorem 2.2. Let $\mathcal{M}$ be a nonempty subset of a q-normed space $\mathcal{X}$ and let $\mathcal{T}, \mathcal{S}: \Omega \times \mathcal{X} \rightarrow \mathcal{X}$ be continuous random operator such that for each $\omega \in \Omega$. Assume that $\mathcal{M}$ has the property $(N)$ with $p \in \mathcal{S}(\omega, p), \mathcal{S}(\omega, \mathcal{M})=\mathcal{M}$ and $\mathcal{T}(\omega, \mathcal{M}-\{p\}) \subseteq \mathcal{S}(\omega, \mathcal{M}-\{p\})$ where $p \in F i x(\mathcal{S})$. Suppose $\mathcal{T}$ is uniformly asymptotically regular, asymptotically $\mathcal{S}$-nonexpansive with sequence $\left\{k_{n}(\omega)\right\}$ and $\mathcal{S}(\omega,$.$) satisfies property (C)$. If $\mathcal{T}, \mathcal{S}$ be uniformly $\mathcal{R}$-subweakly 
commutative random operators on $\mathcal{M}$, then there exists a measurable map $\xi: \Omega \rightarrow \mathcal{M}$ such that $\xi(\omega)=\mathcal{T}(\omega, \xi(\omega))=\mathcal{S}(\omega, \xi(\omega))$ for each $\omega \in \Omega$, if one of the following conditions is satisfied:

(1) $\mathcal{M}$ is compact and $\mathcal{S}$ is continuous;

(2) $\mathcal{X}$ be a complete $q$-normed space whose dual $\mathcal{X}^{\prime}$ separates the points of $\mathcal{X}, \mathcal{M}$ is weakly compact, $\mathcal{S}$ is weakly continuous and $\left(\mathcal{S}-\mathcal{T}^{n}\right)(\omega,$. is demiclosed at 0 ;

(3) $\mathcal{S}$ is weakly continuous, $\mathcal{M}$ is weakly compact and $\mathcal{X}$ be a complete $q$-normed space whose dual $\mathcal{X}^{\prime}$ separates the points of $\mathcal{X}$ satisfying Opial's condition.

Proof. From Theorem 2.1, for each $n \geq 1$, there exists exactly one point in $\mathcal{M}$ such that

$$
\mathcal{S}\left(\omega, \xi_{n}(\omega)\right)=\xi_{n}(\omega)=\mu_{n}(\omega) \mathcal{T}^{n}\left(\omega, \xi_{n}(\omega)\right)+\left(1-\mu_{n}(\omega)\right) p .
$$

Also

$$
\left\|\xi_{n}(\omega)-\mathcal{T}^{n}\left(\omega, \xi_{n}(\omega)\right)\right\|_{q}=\left(1-\mu_{n}(\omega)\right)^{q}\left\|\mathcal{T}^{n}\left(\omega, \xi_{n}(\omega)\right)-p\right\|_{q} .
$$

Since $\mathcal{T}(\mathcal{M}-\{p\})$ is bounded and $k_{n}(\omega) \rightarrow 1$ as $n \rightarrow \infty$, it follows that $\left\|\xi_{n}(\omega)-\mathcal{T}^{n}\left(\omega, \xi_{n}(\omega)\right)\right\|_{q} \rightarrow 0$. Now

$$
\begin{aligned}
& \left\|\xi_{n}(\omega)-\mathcal{T}\left(\omega, \xi_{n}(\omega)\right)\right\|_{q} \leq\left\|\xi_{n}(\omega)-\mathcal{T}^{n}\left(\omega, \xi_{n}(\omega)\right)\right\|_{q} \\
& \quad+\left\|\mathcal{T}^{n}\left(\omega, \xi_{n}(\omega)\right)-\mathcal{T}^{n+1}\left(\omega, \xi_{n}(\omega)\right)\right\|_{q} \\
& \quad+\left\|\mathcal{T}^{n+1}\left(\omega, \xi_{n}(\omega)\right)-\mathcal{T}\left(\omega, \xi_{n}(\omega)\right)\right\|_{q} \\
& \leq\left\|\xi_{n}(\omega)-\mathcal{T}^{n}\left(\omega, \xi_{n}(\omega)\right)\right\|_{q} \\
& \quad+\left\|\mathcal{T}^{n}\left(\omega, \xi_{n}(\omega)\right)-\mathcal{T}^{n+1}\left(\omega, \xi_{n}(\omega)\right)\right\|_{q} \\
& \quad+\left[k_{1}(\omega)\right]^{q}\left\|\mathcal{S}\left(\omega, \mathcal{T}^{n}\left(\omega, \xi_{n}(\omega)\right)\right)-\mathcal{S}\left(\omega, \xi_{n}(\omega)\right)\right\|_{q} .
\end{aligned}
$$

Since $\mathcal{S}$ is continuous, satisfies property $(C)$ and $\mathcal{T}$ is uniformly asymptotically regular, we have

$$
\begin{aligned}
\left\|\xi_{n}(\omega)-\mathcal{T}\left(\omega, \xi_{n}(\omega)\right)\right\|_{q} \leq & \left\|\xi_{n}(\omega)-\mathcal{T}^{n}\left(\omega, \xi_{n}(\omega)\right)\right\|_{q} \\
& +\left\|\mathcal{T}^{n}\left(\omega, \xi_{n}(\omega)\right)-\mathcal{T}^{n+1}\left(\omega, \xi_{n}(\omega)\right)\right\|_{q} \\
& +\left[k_{1}(\omega)\right]^{q}\left\|\mathcal{S}\left(\omega, \mathcal{T}^{n}\left(\omega, \xi_{n}(\omega)\right)\right)-\xi_{n}(\omega)\right\|_{q}
\end{aligned}
$$

as $n \rightarrow \infty$. Thus $\mathcal{T}\left(\omega, \xi_{n}(\omega)\right)-\xi_{n}(\omega) \rightarrow 0$ as $n \rightarrow \infty$.

(1) Since $\mathcal{M}$ is compact, therefore, in the line of Theorem 3.1 [18], there exists a subsequence $\left\{\xi_{m}\right\}$ of $\left\{\xi_{n}\right\}$ such that $\xi_{m}(\omega) \rightarrow \xi(\omega) \in \mathcal{M}$ as $m \rightarrow \infty$. By the continuity of $\mathcal{T}$, we have $\mathcal{T}(\omega, \xi(\omega))=\xi(\omega)$. Since $\mathcal{T}(\mathcal{M}-\{p\}) \subset \mathcal{S}(\mathcal{M}-\{p\})$, it follows that $\xi(\omega)=\mathcal{T}(\omega, \xi(\omega)=$ $\mathcal{S}(\omega, \zeta(\omega))$ for some $\zeta(\omega) \in \mathcal{M}$. Moreover,

$$
\begin{aligned}
\left\|\mathcal{T}\left(\omega, \xi_{m}(\omega)\right)-\mathcal{T}(\omega, \zeta(\omega))\right\|_{q} & \leq\left[k_{1}(\omega)\right]^{q}\left\|\mathcal{S}\left(\omega, \xi_{m}(\omega)\right)-\mathcal{S}(\omega, \zeta(\omega))\right\|_{q} \\
& =\left[k_{1}(\omega)\right]^{q}\left\|\xi_{m}(\omega)-\zeta(\omega)\right\|_{q} .
\end{aligned}
$$


Taking the limit as $m \rightarrow \infty$, we get $\mathcal{T}(\omega, \xi(\omega))=\mathcal{T}(\omega, \zeta(\omega))$. Thus, $\xi(\omega)=\mathcal{T}(\omega, \xi(\omega))=\mathcal{T}(\omega, \zeta(\omega))=\mathcal{S}(\omega, \zeta(\omega))$. Since $\mathcal{S}$ and $\mathcal{T}$ are uniformly $\mathcal{R}$-subweakly commuting on $\mathcal{M}-\{p\}$, it follows that

$$
\begin{aligned}
\|\mathcal{T}(\omega, \xi(\omega))-\mathcal{S}(\omega, \xi(\omega))\|_{q} & =\|\mathcal{T}(\omega, \mathcal{S}(\omega, \zeta(\omega)))-\mathcal{S}(\omega, \mathcal{T}(\omega, \zeta(\omega)))\|_{q} \\
& \leq \mathcal{R}\|\mathcal{T}(\omega, \zeta(\omega))-\mathcal{S}(\omega, \zeta(\omega))\|_{q}=0 .
\end{aligned}
$$

Hence, we have $\mathcal{T}(\omega, \xi(\omega))=\mathcal{S}(\omega, \xi(\omega))=\xi(\omega)$.

(2) Since $\mathcal{M}$ is weakly compact, therefore, in the line of Theorem 3.2 [18], there exists a subsequence $\left\{\xi_{m}\right\}$ of $\left\{\xi_{n}\right\}$ such that $\xi_{m}(\omega) \rightarrow \xi(\omega) \in$ $\mathcal{M}$ as $m \rightarrow \infty$. Now, from weakly continuity of $\mathcal{S}$, we have

$$
\begin{aligned}
\mathcal{S}(\omega, \xi(\omega)) & =\mathcal{S}\left(\omega, \lim _{m \rightarrow \infty} \xi_{m}(\omega)\right)= \\
& =\lim _{m \rightarrow \infty} \mathcal{S}\left(\omega, \xi_{m}(\omega)\right)= \\
& =\lim _{m \rightarrow \infty} \xi_{m}(\omega)=\xi(\omega) .
\end{aligned}
$$

Now,

$$
\begin{aligned}
\mathcal{S}\left(\omega, \xi_{m}(\omega)\right)-\mathcal{T}^{m}\left(\omega, \xi_{m}(\omega)\right) & =\xi_{m}(\omega)-\mathcal{T}^{m}\left(\omega, \xi_{m}(\omega)\right) \\
& =\mathcal{T}_{m}\left(\omega, \xi_{m}(\omega)\right)-\mathcal{T}^{m}\left(\omega, \xi_{m}(\omega)\right) \\
& =\left(1-\mu_{m}(\omega)\right)\left(p-\mathcal{T}^{m}\left(\omega, \xi_{m}(\omega)\right)\right) .
\end{aligned}
$$

Since $\mathcal{M}$ is bounded and $\mu_{m}(\omega) \rightarrow 1$, it follows that

$$
\left\|\mathcal{S}\left(\omega, \xi_{m}(\omega)\right)-\mathcal{T}^{m}\left(\omega, \xi_{m}(\omega)\right)\right\|_{q} \rightarrow 0 .
$$

Since $\left(\mathcal{S}-\mathcal{T}^{m}\right)(\omega,$.$) is demiclosed at 0$, so $\mathcal{S}(\omega, \xi(\omega))=\mathcal{T}^{m}(\omega, \xi(\omega))$ and so, $\mathcal{S}(\omega, \xi(\omega))=\mathcal{T}^{m}(\omega, \xi(\omega))=\xi(\omega)$. It is remaining to show that $\mathcal{T}(\omega, \xi(\omega))=\xi(\omega)$.

$$
\begin{aligned}
\left\|\mathcal{T}(\omega, \xi(\omega))-\mathcal{T}^{m}(\omega, \xi(\omega))\right\|_{q} & =\left\|\mathcal{T}(\omega, \xi(\omega))-\mathcal{T}\left(\omega, \mathcal{T}^{m-1}(\omega, \xi(\omega))\right)\right\|_{q} \\
& \leq\left[k_{1}(\omega)\right]^{q}\left\|\mathcal{S}(\omega, \xi(\omega))-\mathcal{S}\left(\omega, \mathcal{T}^{m-1}(\omega, \xi(\omega))\right)\right\|_{q}, \\
\|\mathcal{T}(\omega, \xi(\omega))-\xi(\omega)\|_{q} & \leq\left[k_{1}(\omega)\right]^{q}\|\xi(\omega)-\mathcal{S}(\omega, \xi(\omega))\|_{q} \\
& =\left[k_{1}(\omega)\right]^{q}\|\xi(\omega)-\xi(\omega)\|_{q}=0 .
\end{aligned}
$$

a contradiction. Hence $\mathcal{T}(\omega, \xi(\omega))=\xi(\omega)$ which implies $\mathcal{T}(\omega, \xi(\omega))=$ $\mathcal{S}(\omega, \xi(\omega))=\xi(\omega)$.

(3) As in $(2), \mathcal{S}(\omega, \xi(\omega))=\xi(\omega)$ and $\left\|\left(\mathcal{S}-\mathcal{T}^{m}\right)\left(\omega, \xi_{m}(\omega)\right)\right\|_{q} \rightarrow 0$ as $m \rightarrow \infty$. If $\mathcal{S}(\omega, \xi(\omega)) \neq \mathcal{T}^{m}(\omega, \xi(\omega))$, then by Opial's condition of $\mathcal{X}$ and asymptotically $\mathcal{S}$-nonexpansiveness of $\mathcal{T}$, it follows that 


$$
\begin{aligned}
& \liminf _{m \rightarrow \infty}\left\|\mathcal{S}\left(\omega, \xi_{m}(\omega)\right)-\mathcal{S}(\omega, \xi(\omega))\right\|_{q} \\
< & \liminf _{m \rightarrow \infty}\left\|\mathcal{S}\left(\omega, \xi_{m}(\omega)\right)-\mathcal{T}^{m}(\omega, \xi(\omega))\right\|_{q} \\
< & \liminf _{m \rightarrow \infty}\left\|\mathcal{S}\left(\omega, \xi_{m}(\omega)\right)-\mathcal{T}^{m}\left(\omega, \xi_{m}(\omega)\right)\right\|_{q} \\
& \quad+\liminf _{m \rightarrow \infty}\left\|\mathcal{T}^{m}\left(\omega, \xi_{m}(\omega)\right)-\mathcal{T}^{m}(\omega, \xi(\omega))\right\|_{q} \\
< & \liminf _{m \rightarrow \infty}\left\|\mathcal{T}^{m}\left(\omega, \xi_{m}(\omega)\right)-\mathcal{T}^{m}(\omega, \xi(\omega))\right\|_{q} \\
\leq & {\left[k_{m}(\omega)\right]^{q}\left\|\mathcal{S}\left(\omega, \xi_{m}(\omega)\right)-\mathcal{S}(\omega, \xi(\omega))\right\|_{q} }
\end{aligned}
$$

a contradiction. Hence $\mathcal{S}(\omega, \xi(\omega))=\mathcal{T}^{m}(\omega, \xi(\omega))=\xi(\omega)$. We can show that $\mathcal{T}(\omega, \xi(\omega))=\mathcal{S}(\omega, \xi(\omega))$ as in (2).

Following are the extension of Theorem 3.6 and Theorem 3.7 [18] in the setting of domain which is not necessarily non-starshaped and relaxing the linearity of random operator $\mathcal{S}$ respectively:

Theorem 2.3. Let $\mathcal{X}$ be a q-normed space. Let $\mathcal{T}, \mathcal{S}: \Omega \times \mathcal{X} \rightarrow \mathcal{X}$ be continuous random operators and $\mathcal{M} \subseteq \mathcal{X}$ such that $\mathcal{T}(\omega,):. \partial \mathcal{M} \cap \mathcal{M} \rightarrow$ $\mathcal{M}$, where $\partial \mathcal{M}$ stands for the boundary of $\mathcal{M}$. Let $x_{0}=\mathcal{T}\left(\omega, x_{0}\right)=\mathcal{S}\left(\omega, x_{0}\right)$ for each $x_{0} \in \mathcal{X}$ and $\omega \in \Omega$. Suppose $\mathcal{T}$ is uniformly asymptotically regular, asymptotically $\mathcal{S}$-nonexpansive and $\mathcal{S}(\omega,$.$) satisfies property (C)$ on $\mathcal{P}_{\mathcal{M}}\left(x_{0}\right)$ with $\mathcal{S}\left(\omega, \mathcal{P}_{\mathcal{M}}\left(x_{0}\right)\right)=\mathcal{P}_{\mathcal{M}}\left(x_{0}\right)$. If $\mathcal{P}_{\mathcal{M}}\left(x_{0}\right)$ is nonempty, has the property $(N)$ with $p \in \mathcal{S}(\omega, p)$ and $\mathcal{T}$ and $\mathcal{S}$ are uniformly $\mathcal{R}$-subweakly commuting mappings on $\mathcal{P}_{\mathcal{M}}\left(x_{0}\right) \cup\left\{x_{0}\right\}$ satisfying $\left\|\mathcal{T}(\omega, x)-\mathcal{T}\left(\omega, x_{0}\right)\right\|_{q} \leq \| \mathcal{S}(\omega, x)-$ $\mathcal{S}\left(\omega, x_{0}\right) \|_{q}$, then there exists a measurable map $\xi: \Omega \rightarrow \mathcal{P}_{\mathcal{M}}\left(x_{0}\right)$ such that $\xi(\omega)=\mathcal{T}(\omega, \xi(\omega))=\mathcal{S}(\omega, \xi(\omega))$ for each $\omega \in \Omega$, if one of the following conditions is satisfied:

(1) $\mathcal{P}_{\mathcal{M}}\left(x_{0}\right)$ is compact and $\mathcal{S}$ is continuous;

(2) $\mathcal{X}$ be a complete $q$-normed space whose dual $\mathcal{X}^{\prime}$ separates the points of $\mathcal{X}, \mathcal{P}_{\mathcal{M}}\left(x_{0}\right)$ is weakly compact, $\mathcal{S}$ is weakly continuous and $(\mathcal{S}-$ $\left.\mathcal{T}^{n}\right)(\omega,$.$) is demiclosed at 0$;

(3) $\mathcal{S}$ is weakly continuous, $\mathcal{P}_{\mathcal{M}}\left(x_{0}\right)$ is weakly compact and $\mathcal{X}$ be a complete $q$-normed space whose dual $\mathcal{X}^{\prime}$ separates the points of $\mathcal{X}$ satisfying Opial's condition.

Proof. Let $y \in \mathcal{P}_{\mathcal{M}}\left(x_{0}\right)$. Then $\left\|y-x_{0}\right\|_{q}=\operatorname{dist}(x, \mathcal{M})$. Note that for any $t(\omega) \in(0,1)$,

$$
\left\|t(\omega) x_{0}+(1-t(\omega)) y-x_{0}\right\|_{q}=(1-t(\omega))^{q}\left\|y-x_{0}\right\|_{q}<\operatorname{dist}\left(x_{0}, \mathcal{M}\right) .
$$

It follows that the line segment $\left\{t(\omega) x_{0}+(1-t(\omega)) y: 0<t(\omega)<1\right\}$ and the set $\mathcal{M}$ are disjoint. Thus $y$ is not in the interior of $\mathcal{M}$ and so $y \in \partial \mathcal{M} \cap \mathcal{M}$. Since $\mathcal{T}(\partial \mathcal{M} \cap \mathcal{M}) \subset \mathcal{M}, \mathcal{T} x$ must be in $\mathcal{M}$. Also since $\mathcal{S}(\omega, y) \in \mathcal{P}_{\mathcal{M}}\left(x_{0}\right)$, $x_{0}=\mathcal{T}\left(\omega, x_{0}\right)=\mathcal{S}\left(\omega, x_{0}\right)$ and therefore by the given contractive condition, 
we have

$$
\begin{aligned}
\left\|\mathcal{T}(\omega, y)-x_{0}\right\|_{q} & =\left\|\mathcal{T}(\omega, y)-\mathcal{T}\left(\omega, x_{0}\right)\right\|_{q} \leq \\
& \leq\left\|\mathcal{S}(\omega, x)-\mathcal{S}\left(\omega, x_{0}\right)\right\|_{q}= \\
& =\left\|\mathcal{S}(\omega, y)-x_{0}\right\|_{q}=\operatorname{dist}\left(x_{0}, \mathcal{M}\right)
\end{aligned}
$$

Consequently $\mathcal{P}_{\mathcal{M}}\left(x_{0}\right)$ is $\mathcal{T}(\omega,$.$) -invariant. Hence,$

$$
\mathcal{T}\left(\omega, \mathcal{P}_{\mathcal{M}}\left(x_{0}\right)\right) \subseteq \mathcal{P}_{\mathcal{M}}\left(x_{0}\right)=\mathcal{S}\left(\omega, \mathcal{P}_{\mathcal{M}}\left(x_{0}\right)\right)
$$

Thus, the result follows from Theorem 2.2.

Define $\mathcal{C}_{\mathcal{M}}^{\mathcal{S}}\left(x_{0}\right)=\left\{x \in \mathcal{M}: \mathcal{S} x \in \mathcal{P}_{\mathcal{M}}\left(x_{0}\right)\right\}$ and $\mathcal{D}_{\mathcal{M}}^{\mathcal{S}}\left(x_{0}\right)=\mathcal{P}_{\mathcal{M}}\left(x_{0}\right) \cap$ $\mathcal{C}_{\mathcal{M}}^{\mathcal{S}}\left(x_{0}\right)[1]$

Theorem 2.4. Let $\mathcal{X}$ be a q-normed space. Let $\mathcal{T}, \mathcal{S}: \Omega \times \mathcal{X} \rightarrow \mathcal{X}$ be random operators and $\mathcal{M} \subseteq \mathcal{X}$ such that $\mathcal{T}(\omega,):. \partial \mathcal{M} \rightarrow \mathcal{M}$, where $\partial \mathcal{M}$ stands for the boundary of $\mathcal{M}$. Let $x_{0}=\mathcal{T}\left(\omega, x_{0}\right)=\mathcal{S}\left(\omega, x_{0}\right)$ for each $x_{0} \in$ $\mathcal{X}$ and $\omega \in \Omega$. Suppose $\mathcal{T}$ is continuous, uniformly asymptotically regular, asymptotically $\mathcal{S}$-nonexpansive and $\mathcal{S}\left(\omega\right.$, .) be nonexpansive $\mathcal{P}_{\mathcal{M}}\left(x_{0}\right) \cup\left\{x_{0}\right\}$ and satisfies property $(C)$ on $\mathcal{D}=\mathcal{D}_{\mathcal{M}}^{\mathcal{S}}\left(x_{0}\right)$ with $\mathcal{S}(\omega, \mathcal{D})=\mathcal{D}$. If $\mathcal{D}$ is nonempty, has the property $(N)$ with $p \in \mathcal{S}(\omega, p)$ and and $\mathcal{T}$ and $\mathcal{S}$ are uniformly $\mathcal{R}$-subweakly commuting mappings on $\mathcal{P}_{\mathcal{M}}\left(x_{0}\right) \cup\left\{x_{0}\right\}$ satisfying $\left\|\mathcal{T}(\omega, x)-\mathcal{T}\left(\omega, x_{0}\right)\right\|_{q} \leq\left\|\mathcal{S}(\omega, x)-\mathcal{S}\left(\omega, x_{0}\right)\right\|_{q}$, then there exists a measurable map $\xi: \Omega \rightarrow \mathcal{P}_{\mathcal{M}}\left(x_{0}\right)$ such that $\xi(\omega)=\mathcal{T}(\omega, \xi(\omega))=\mathcal{S}(\omega, \xi(\omega))$ for each $\omega \in \Omega$ if one of the following conditions is satisfied:

(1) $\mathcal{D}$ is compact and $\mathcal{S}$ is continuous;

(2) $\mathcal{X}$ be a complete $q$-normed space whose dual $\mathcal{X}^{\prime}$ separates the points of $\mathcal{X}, \mathcal{D}$ is weakly compact, $\mathcal{S}$ is weakly continuous and $\left(\mathcal{S}-\mathcal{T}^{n}\right)(\omega,$. is demiclosed at 0 ;

(3) $\mathcal{S}$ is weakly continuous, $\mathcal{D}$ is weakly compact and $\mathcal{X}$ be a complete $q$-normed space whose dual $\mathcal{X}^{\prime}$ separates the points of $\mathcal{X}$ satisfying Opial's condition.

Proof. Let $y \in \mathcal{D}$, then $\mathcal{S}(\omega, y) \in \mathcal{D}$, since $\mathcal{S}(\omega, \mathcal{D})=\mathcal{D}$ for each $\omega \in \Omega$. Also, if $y \in \partial \mathcal{M}$ and so $\mathcal{T}(\omega, y) \in \mathcal{M}$, since $\mathcal{T}(\omega, \partial \mathcal{M}) \subseteq \mathcal{M}$ for each $\omega \in \Omega$. Now since $x_{0}=\mathcal{T}\left(\omega, x_{0}\right)$ and $\mathcal{T}$ is $\mathcal{S}$-nonexpansive map, we have

$$
\left\|\mathcal{T}(\omega, y)-x_{0}\right\|_{q}=\left\|\mathcal{T}(\omega, y)-\mathcal{T}\left(\omega, x_{0}\right)\right\|_{q} \leq\left\|\mathcal{S}(\omega, y)-\mathcal{S}\left(\omega, x_{0}\right)\right\|_{q}
$$

As $\mathcal{S}\left(\omega, x_{0}\right)=x_{0}$, we therefore have,

$$
\left\|\mathcal{T}(\omega, y)-x_{0}\right\|_{q} \leq\left\|\mathcal{S}\left(\omega, x_{0}\right)-x_{0}\right\|_{q}=\operatorname{dist}\left(x_{0}, \mathcal{M}\right),
$$

since $\mathcal{S}(\omega, y) \in \mathcal{P}_{\mathcal{M}}\left(x_{0}\right)$. This implies that $\mathcal{T}(\omega, y)$ is also closest to $x_{0}$, so, $\mathcal{T}(\omega, y) \in \mathcal{P}_{\mathcal{M}}\left(x_{0}\right)$; consequently $\mathcal{P}_{\mathcal{M}}\left(x_{0}\right)$ is $\mathcal{T}(\omega,$.$) -invariant, that is,$ $\mathcal{T}(\omega,.) \subseteq \mathcal{P}_{\mathcal{M}}\left(x_{0}\right)$. As $\mathcal{S}$ is nonexpansive on $\mathcal{P}_{\mathcal{M}}\left(x_{0}\right) \cup\left\{x_{0}\right\}$, so for each 
$\omega \in \Omega$, we have

$$
\begin{aligned}
\left\|\mathcal{S}(\omega, \mathcal{T}(\omega, y))-x_{0}\right\|_{q} & =\left\|\mathcal{S}(\omega, \mathcal{T}(\omega, y))-\mathcal{S}\left(\omega, x_{0}\right)\right\|_{q} \leq\left\|\mathcal{T}(\omega, y)-x_{0}\right\|_{q} \\
& =\left\|\mathcal{T}(\omega, y)-\mathcal{T}\left(\omega, x_{0}\right)\right\|_{q} \leq\left\|\mathcal{S}(\omega, y)-\mathcal{S}\left(\omega, x_{0}\right)\right\|_{q} \\
& =\left\|\mathcal{S}(\omega, y)-x_{0}\right\|_{q} .
\end{aligned}
$$

Thus, $\mathcal{S}(\omega, \mathcal{T}(\omega, y)) \in \mathcal{P}_{\mathcal{M}}\left(x_{0}\right)$. This implies that $\mathcal{T}(\omega, y) \in \mathcal{C}_{\mathcal{M}}^{\mathcal{S}}\left(x_{0}\right)$ and hence $\mathcal{T}(\omega, y) \in \mathcal{D}$. So, $\mathcal{T}(\omega,$.$) and \mathcal{S}(\omega,$.$) are self-maps on \mathcal{D}$. Hence, all the condition of the Theorem 2.2 are satisfied. Thus, there exists a measurable map $\xi: \Omega \rightarrow \mathcal{D}$ such that $\xi(\omega)=\mathcal{T}(\omega, \xi(\omega))=\mathcal{S}(\omega, \xi(\omega))$ for each $\omega \in \Omega$.

\section{REFERENCES}

[1] M.A. Al-Thagafi, Common fixed points and best approximation, J. Approx. Theory, Vol. 85 (1996), 318-320.

[2] I. Beg, D.R. Sahu and S.D. Diwan, Approximation of fixed points of uniformly R-subweakly commuting mappings, J. Math. Anal. Appl., Vol. 324, No. 2 (2006), 1105-1114.

[3] I. Beg and N. Shahzad, Random fixed points and approximations in random convex metric spaces, J. Appl. Math. Stoch. Anal., Vol. 6 (1993), 237-246.

[4] I. Beg and N. Shahzad, Random fixed points of random multivalued operators on polish spaces, Nonlinear Anal., Vol. 20 (1993), 835-847.

[5] I. Beg and N. Shahzad, Applications of the proximity map to Random fixed points theorems in Hilbert space, J. Math. Anal. Appl., Vol. 196 (1995), 606-613.

[6] I. Beg and N. Shahzad, On invariant random approximations, Approx. Theory Appl., Vol. 12 (1996), 68-72.

[7] I. Beg and N. Shahzad, Common random fixed points of noncommuting random operrators, Random Oper. Stoch. Equ., Vol. 7 (1999), 367-372.

[8] I. Beg and N. Shahzad, An application of a random fixed point theorem to random best approximation, Arch. Math., Vol. 74 (2000), 298-301.

[9] A.T. Bharucha-Reid, Fixed point theorem in probabilistic analysis, Bull. Amer. Math. Soc., Vol. 82 (1976), 641-645.

[10] W.G. Dotson, Fixed point theorems for nonexpasive mappings on starshaped subsets of Banach space, J. London Math. Soc., Vol. 4, No. 2 (1972), 408-410.

[11] C.J. Himmerberg, Measurable relations, Fund. Math., Vol. 87 (1975), 53-72.

[12] N. Hussain, D. O'Regan and R.P. Agarwal, Common fixed point and invariant approximation results on non-starshaped domain, Georgian Math. J., Vol. 12, No. 4 (2005), 559-569.

[13] G. Köthe, Topological vector spaces I, Springer-Verlag, Berlin, 1969.

[14] K. Kuratoski and C. Ryll-Nardzewski, A general theorem on selectors, Bull Acad. Pol. Sci. Ser. Sci. Math. Astron. Phys., Vol. 13 (1965), 397-403. 
[15] T.C. Lin, Random approximations and random fixed point theorems for nonsself maps, Proc. Amer. Math. Soc., Vol. 103 (1988), 1129-1135.

[16] G. Meinardus, Invarianze bei Linearen Approximationen, Arch. Rational Mech. Anal., Vol. 14 (1963), 301-303.

[17] H.K. Nashine, Existence of random best approximation for noncommutative maps, African Diaspora J. Math., Vol. 4, No. 1 (2007), 47-53.

[18] H.K. Nashine, Existence of common random fixed point and random best approximation for non-commuting random operators, Bull. Inst. Math. Acad. Sinica, Vol. 5, No. 1 (20100), 25-40.

[19] N.S. Papagergiou, Random fixed point theorems for measurable multifunctions in Banach spaces, Proc. Amer. Math. Soc., Vol. 97 (1986), 507-514.

[20] N.S. Papagergiou, Random fixed points and random differential inclusions, Internat. J. Math. Math. Sci., Vol. 11 (1988), 551-560.

[21] W. Rudin, Functional Analysis, 2nd ed., International Series in Pure and Applied Mathematics, McGraw-Hill, New York, 1991.

[22] S.A. Sahab, M.S. Khan and S. Sessa, A result in best approximation theory, J. Approx. Theory, Vol. 55 (1988), 349-351.

[23] N. Shahzad, Invariant approximations and R-subweakly commuting maps, J. Math. Anal. Appl., Vol. 257 (2001), 39-45.

[24] S.P. Singh, An application of a fixed point theorem to approximation theory, J. Approx. Theory, Vol. 25 (1979), 89-90.

[25] K.K. Tan and X.Z. Yuan, Random fixed point theorems and approximation in cones, J. Math. Anal. Appl., Vol. 185 (1994), 378-390.

Hemant Kumar Nashine

Department of Mathematics

Disha Institute of Management and Technology

Satya Vihar, Vidhansabha - Chandrakhuri Marg

(Baloda Bazar Road), Mandir Hasaud

RAIPUR-492101 (CHHATTISGARH)

INDIA

E-mail address: hnashine@rediffmail.com

hemantnashine@rediffmail.com 